\title{
Studies for high energy air shower identification using RF measurements with the ASTRONEU array
}

\author{
Stavros Nonis ${ }^{1, *}$, George Bourlis ${ }^{2}$, Ioannis Gkialas ${ }^{1}$, Antonios Leisos ${ }^{2}$, Ioannis Manthos ${ }^{3}$, Kostas Papageorgiou ${ }^{1}$, Apos- \\ tolos Tsirigotis $^{2}$, and Spyros Tzamarias ${ }^{3}$ \\ ${ }^{1}$ Department of Financial and Management Engineering, University of the Aegean, Chios, Greece \\ ${ }^{2}$ Physics Laboratory, School of Science and Technology, Hellenic Open University, Patras, Greece \\ ${ }^{3}$ Department of Physics, Aristotle University of Thessaloniki, Thessaloniki, Greece
}

\begin{abstract}
The Hellenic Open University (HOU) Cosmic Ray Telescope (ASTRONEU) consists of 9 large scintillator detectors and $3 \mathrm{RF}$ antennas arranged in three autonomous stations operating at the Hellenic Open University campus in the city of Patras. High energy showers that are detected simultaneously by two distant stations and in coincidence with the RF antennas are used to study the RF signature of cosmic events. In previous studies we have shown that the timing of the RF signals as well as the measured electric field at the antennas position are in very good agreement with the simulation predictions. In this work we concentrate on the transfer functions of the antennas which are strongly frequency and angular dependent. We show that the RF spectra (at frequencies 30-80 MHz) of the detected showers are exhibiting features of the antenna response as predicted by detailed Monte Carlo simulation suggesting that a single antenna RF spectrum gives access to the cosmic ray arrival direction.
\end{abstract}

\section{Introduction}

High energy cosmic particles hitting the atmospheric molecules produce cascades of secondary particles also known as atmospheric air showers. The (constituents) secondary particles of the air shower are traditionally detected by large arrays of particle detectors (mostly used scintillation detectors and water-Cherenkov detectors). Additionally, the electrons and positrons of very high energy showers are radiating a measurable electric field in the radio frequency (RF) regime. Since a number of experiments have studied the RF signal [1-5] the dominant emission mechanisms are now well understood. The two proposed mechanisms that explain the RF emission are the transverse current variation (opposite deflection of e- and e+ in the earth's magnetic field) [6], and charge excess variation (due to excess of electrons in the shower front) [7]. Among shower's detection methods the radio technique is relatively low cost and is working during daytime and bad weather.

The ASTRONEU cosmic ray telescope is a hybrid Extensive Air Shower (EAS) detection array installed at the Hellenic Open University campus on the outskirts of the city of Patras, Greece. The telescope's layout is divided into 3 autonomous stations each comprising 3 large $\left(\approx 1 \mathrm{~m}^{2}\right)$ scintillator detectors and $1 \mathrm{RF}$ antenna. The array operates in urban environment with strong human made noise collecting data since the beginning of 2014. In previous studies [8] we have shown that it is possible to detect RF signals from showers in noisy environments by

*e-mail: snonis@aegean.gr imposing the appropriate selection criteria to the RF signals. We have also studied the timing and the amplitude strength of the RF signals by comparing the antenna data with the particle detector data as well as with the simulation predictions [9]. In this work we present a detailed Voltage Response Model (VRM) for the RF system using the antenna's Vector Effective Length (VEL) [10] and the electric field at the antenna's position in order to estimate the primary particle arrival direction.

In Section 2 we describe briefly the architecture of the Astroneu array while, in Section 3, the event selection criteria are discussed. In Section 4 we focus on the antenna's response model. We compare the measured RF spectra of the antennas with the VRM predictions, estimate the primary particle arrival direction and compare with the respective direction estimated from the particle detectors. Finally, in Section 5 the conclusions are drawn.

\section{The ASTRONEU Array}

The layout of the stations of the Astoneu array is depicted in figure 1. Each station consists of three HELYCON ${ }^{1}$ Detector Modules (HDM) [12] along with one ${ }^{2}$ RF Antenna (RFA) [10] and is equipped with trigger, digitization and Data Acquisition (DAQ) electronics. It is also equipped with slow control and monitor electronics and a GPS-based timing system.

\footnotetext{
${ }^{1}$ HELYCON is the acronym of Hellenic Lyceum Cosmic Observatories Network a project aiming for the development of a network of extensive air shower detector stations, distributed in western Greece [11].

${ }^{2}$ In 2017 three more RF antennas were installed at station A.
} 


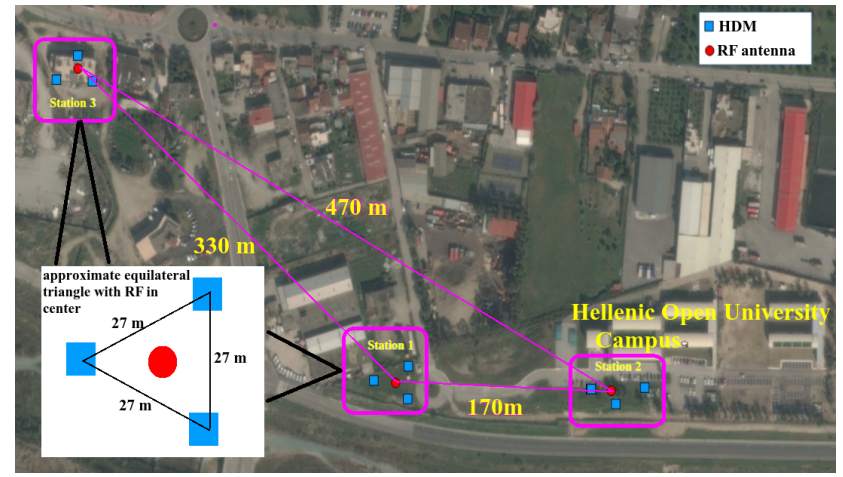

Figure 1. The outline of the ASTRONEU array installed at the HOU campus. Each of the three stations comprises three Helycon Detector Modules and a CODALEMA type RF antenna. Picture taken from [13].

The HDM is made of 160 scintillating tiles covering an area of about $1 \mathrm{~m}^{2}$. The light generated by the interaction of shower particles with the scintillation material, is collected by wavelength shifting fibers embedded in the scintillator material and is driven to a photomultiplier tube (PMT). The RF antenna is a "Butterfly" bowtie antenna [10] developed by the CODALEMA collaboration [1] with dimension $2 \times 2 \times 1 \mathrm{~m}^{3}$ constructed with two electrically short dipoles aligned in the East-West (EW) and North-South (NS) directions. The dipole signals are fed directly into the input of a Low Noise Amplifier (LNA) [14] at the center of the antenna and they are sampled at a rate of $1 \mathrm{GHz}$.

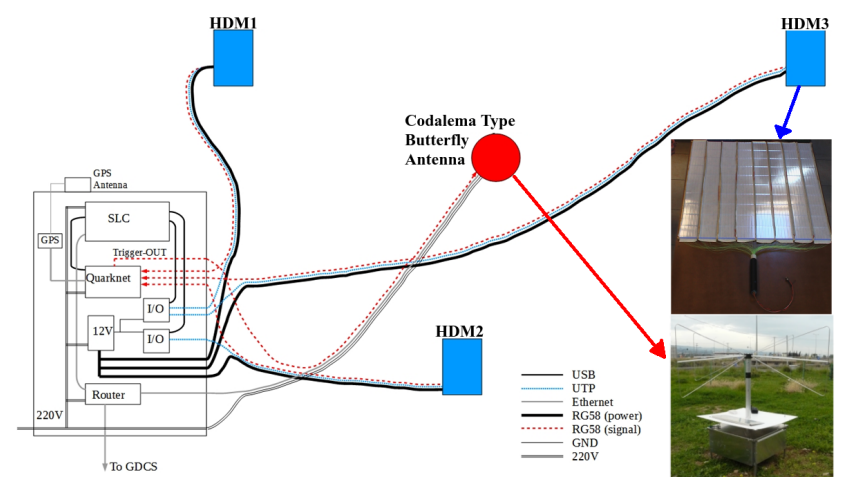

Figure 2. Schematic representation of the connections in the Astroneu station. Picture taken from [13].

A schematic representation of the Astroneu station architecture is shown in figure 2. The signals of the three HDM PMTs of a station are readout by a Quarknet electronics board [15] which measures with $1.25 \mathrm{~ns}$ accuracy the time of the crossings of the PMT waveforms with a predefined voltage threshold. The time of the first crossing defines the timing of the pulse, while the time that the pulse remains above the threshold (Time over Threshold ToT) is used to estimate the pulse size. The necessary I/O and network devices as well as the Station Local Computer (SLC) that communicates with the RFA-DAQ and hosts the Quarknet-DAQ and the monitor/control software of the station are located inside a metallic Central Electronics Box (CEB). A station trigger is generated when all three HDMs of the station acquire signals above $9.7 \mathrm{mV}$ in a time window of $240 \mathrm{~ns}$. This trigger signal is driven into the RFA external trigger input which activates the recording of 2560 samples of each waveform in the two poles of the antenna. The experimental information from both the HDMs and the RFA of a station are transferred to the Global Data and Control Server (GDCS), where the event building is performed offline utilising the time-tags of the data provided by GPS devices embedded in the corresponding DAQs.

The detector network has been operated, collecting data since August 2014. Standard installation, calibration and operation procedures have been established [12] and software packages to simulate in detail the response of the detectors to showers as well as to reconstruct the EAS characteristics have been developed [13]. Each autonomous station of the Astroneu array is capable of reconstructing extensive air showers of energy more than $10 \mathrm{TeV}$ with a typical resolution of 3.5 degrees at a rate of $17 h^{-1}$. The performance of the Astroneu array in detecting and reconstructing EAS using the charged particle detectors of a station (single station operation) or by combining the experimental information from two stations (multiple station operation) is reported in [13]. The RF component of the EAS has been studied using noise filters, timing and signal polarization [8]. Further studies including the correlation of the RF signals with the particle detector data as well as the comparison of the electric field measurements with the MC prediction have also been reported [9].

\section{Data Selection}

The data sample used in this analysis was collected by stations A and B (see figure 1) in coincidence, from 2014 August 1st to 2016 March 16th. Detailed MC studies [13] show that these double station events correspond to EAS energies $E>5 \cdot 10^{3} \mathrm{TeV}$. Each station was triggered when all three HDM signals were above $9.7 \mathrm{mV}$ (corresponding roughly to 2 minimum ionizing particles), while coincidence events between stations were identified offline using the event GPS time and the distance between the stations $(164 \mathrm{~m})$. The final sample after passing all the quality criteria consisted of 1395 events corresponding to an event rate of about $0.15 h^{-1}$. For each event, the ToT value and the timing of the six HDM pulses as well as the full waveform of each pole of the two RF antennas were kept for further analysis.

For each reconstructed shower, the RF signals were filtered in order to keep frequencies in the range $20-$ $80 \mathrm{MHz}$, since for frequencies below $20 \mathrm{MHz}$ the ionosphere is strongly reflective, and human made signals from long distances can still contribute to the noise, while at high frequencies, over $80 \mathrm{MHz}$, strong signals from the radio FM band are present. The filtering procedure is implemented in two phases. Initially, the waveform is subject to Tukey filtering, suppressing the outliers, and then, a 

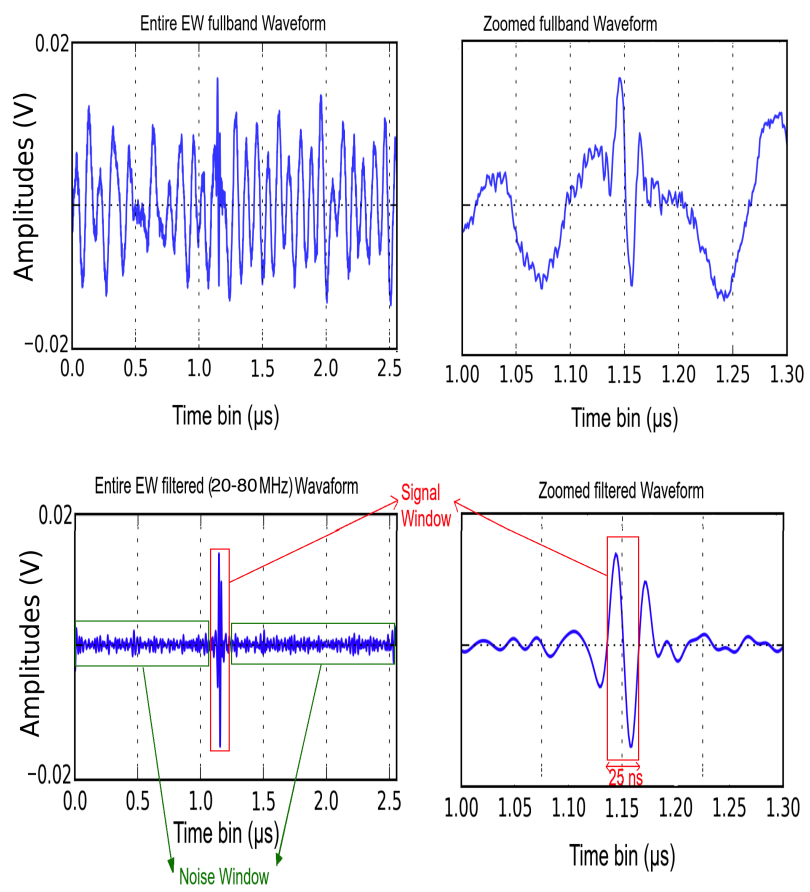

Figure 3. The upper plots show the raw EW waveform of a cosmic event. The lower plots show the corresponding filtered signal in the $20-80 \mathrm{MHz}$ band. In both cases the full waveform (left) and the central region (right) are presented.

Fast Fourier Transform (FFT) is applied. After this transformation, the non-desirable frequencies are removed and subsequently the inverse Fourier Transform $\left(\mathrm{FFT}^{-1}\right)$ is applied, providing the filtered signal (figure 3 ).

The filtered RF waveforms of the data sample were retained for further analysis when a peak value of at least 10 times above the noise was observed in a time window of $100 \mathrm{~ns}$ around the trigger signal (signal time window). The noise was estimated as the $\mathrm{rms}^{3}$ of the voltage in a large time window exluding the signal time window. Additionally, in order to select very high energy showers another criterion based on the particle detectors information was applied. As it is reported in [9] the sum of the ToT values of the $6 \mathrm{HDMs}$ of stations A and B over the projected HDM area perpendicular to the shower axis is a measurement of the ground particle density and can be used to select showers of higher energy. In this analysis a threshold value of $500 \mathrm{~ns}$ was used and the final sample consisted of 34 events from Station-A and 48 events from Station-B. In this data sample the EW component of the RF signal was used to define the timing of the RF signal evaluated as the time instance of the peak value of the filtered waveform. The timing of the RF signal is found to be consistent [9] with the plane particle front approximation, the timing of the HDM pulses and the geometry of the station ${ }^{4}$. Furthermore, the strength of the RF signals were used to estimate the electric field intensity at the antennas position

\footnotetext{
${ }^{3}$ Root of the Mean of the Square

${ }^{4}$ Using the direction of the shower axis as estimated by the particle detectors and the position of the antenna the expected delay of the RF signal w.r.t. an HDM signal can be evaluated with triangulation.
}

and found to be in very good agreement with the MC prediction [9].

\section{Cosmic Ray Arrival Reconstruction from Single RF Antenna Spectrum}

\subsection{Vector Effective Length Evaluation}

The gain $G$ of an antenna is a parameter which takes into account the receiving efficiency (as well as the radiation efficiency) of the antenna and its directional dependencies. It also characterizes the frequency dependence of the structure. In the majority of modern cosmic rays RF detection experiments, the antennas are connected to a Low Noise Amplifier (LNA). Thus a more detailed description of the directional and frequency dependencies of the setup (antenna+LNA) is achieved using the Vector effective Length (VEL) (or alternative known as system transfer functions) [1]. The VEL is considered to be the ratio of the voltage that appears at the LNA output $(V)$ to the magnitude of the electric-field strength $(E)$ in the direction of the antenna polarization $(V E L=V / E)$.

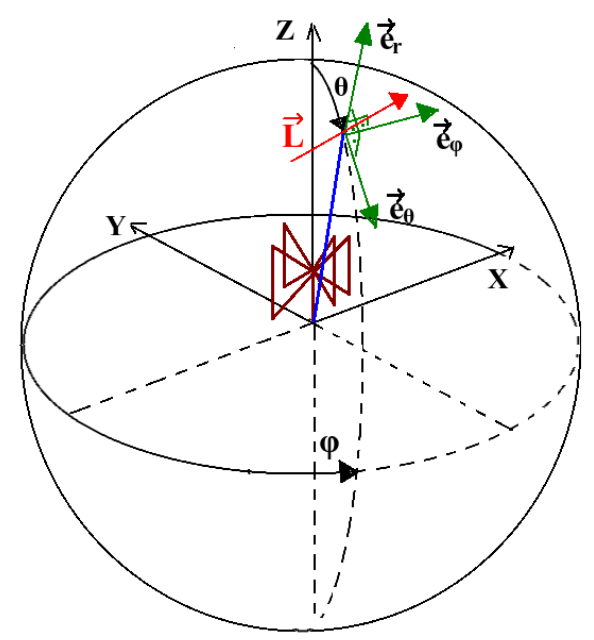

Figure 4. The spherical coordinate system with the antenna in the center. The zenith angle $\theta$ is counted from the top, the azimuth angle $\phi$ from the x-axis (along NS direction) of the coordinate system. The RF signal arrives from a specific direction $(\theta, \phi)$. The VEL $\vec{L}$ for the specified direction is shown.

Let's consider a spherical coordinate system with the antenna in its center as shown in figure 4. In this coordinate system, the electric field of a plane wave arrives from a given direction $(\theta, \phi)$. The antenna's VEL, for each pole separately, can be expressed with a two-component vector along the directions of the unit vectors $\overrightarrow{e_{\theta}}$ and $\overrightarrow{e_{\phi}}$,

$$
\vec{L}_{e w / n s}(\theta, \phi, t)=L_{e w / n s}(\theta, t) \cdot \overrightarrow{e_{\theta}}+L_{e w / n s}(\phi, t) \cdot \overrightarrow{e_{\phi}}
$$

The antenna characteristics contained in VEL are usually obtained in the frequency domain rather than the time domain as provided by the Fourier transforms $\mathcal{F}$ of the quantities

$$
\vec{L}_{e w / n s}(\theta, \phi, f)=\mathcal{F}\left(L_{e w / n s}(\theta, t)\right) \cdot \overrightarrow{e_{\theta}}+\mathcal{F}\left(L_{e w / n s}(\phi, t)\right) \cdot \overrightarrow{e_{\phi}}
$$


The VEL's amplitude can be described in terms of the gain $G(\theta, \phi)$ and structural features of the system such as the antenna radiation resistance and reactance $\left(R_{\text {rad }}, X_{\text {ant }}\right)$ and the LNA input resistance and reactance $\left(R_{L N A}, X_{L N A}\right)$ according to $[16,17]$

$$
L_{e w / n s}(\theta, \phi, f)=\frac{c}{f} \sqrt{\frac{G_{e w / n s}(\theta, \phi)}{\pi Z_{0}}} \cdot \frac{\sqrt{R_{\text {rad }}}\left|R_{L N A}+i X_{L N A}\right|}{\left|R_{L N A}+i X_{L N A}+R_{r a d}+i X_{a n t}\right|}
$$

with $Z_{0}$ the intrinsic impedance and $f$ the frequency of the received electric field.

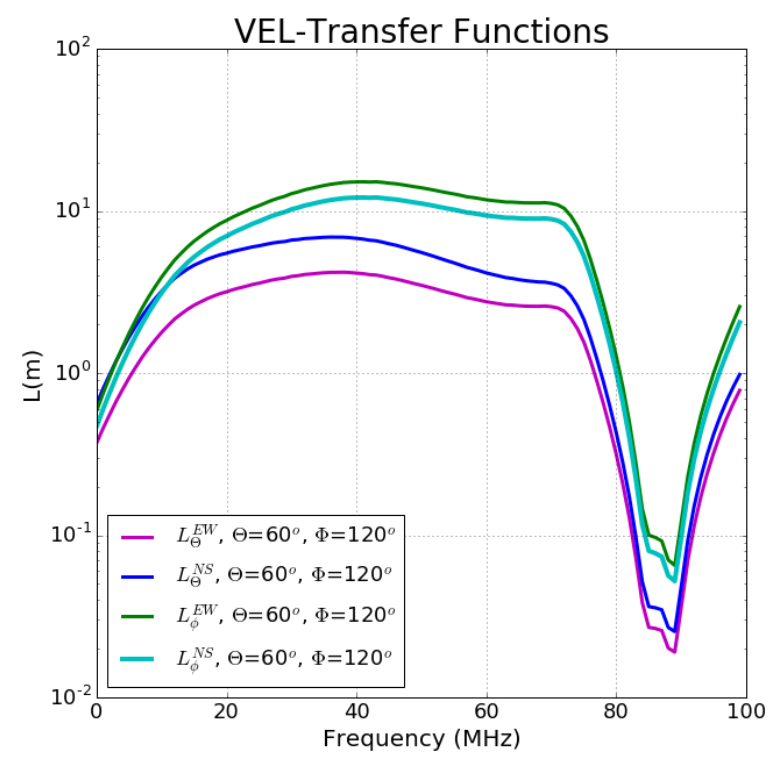

Figure 5. Butterfly Antenna VEL $30-80 \mathrm{MHz}$ for EW/NS poles and for direction $\theta=60^{\circ}, \phi=120^{\circ}$.

The evaluation of the VEL of each pole is performed utilizing the NEC software [18]. An example is shown in figure 5 where the two components of the effective length of each pole are evaluated as a function of the frequency $(0-100 \mathrm{MHz})$ for a signal arriving direction $\theta=60^{\circ}$ and $\phi=120^{\circ}$.

\subsection{Voltage Response Model}

The voltage magnitude (response) of the system to an incident electric field that arrives from a given direction $(\theta, \phi)$ (in a single polarization) is obtained by the convolution of the field and the VEL of the antenna [19]. For the two antenna's poles the response can be written in time domain [20] as follows

$$
V_{e w / n s}(\theta, \phi, t)=\vec{L}_{e w / n s}(\theta, \phi, t) * \vec{E}_{e w / n s}(\theta, \phi, t)
$$

In the frequency domain the antenna respone can be expressed with multiplication of their Fourier transforms (according to convolution theorem [17])

$$
V_{e w / n s}(\theta, \phi, f)=\vec{L}_{e w / n s}(\theta, \phi, f) \cdot \vec{E}_{e w / n s}(\theta, \phi, f)
$$

For the calculation of the electric field the SELFAS [21] simulation package is used. SELFAS calculates the $\mathrm{RF}$ signal, in the $\mathrm{MHz}$ range, emitted by cosmic ray air showers during their formation in the atmosphere. The simulation includes RF emission by charge excess variation (due to excess of electrons in the shower front) and by transverse current variation (due to the opposite direction deflections of $e^{-}$and $e^{+}$in the earth magnetic field). Based on "shower universality", it generates only $e^{+}$and $e^{-}$in the shower front according to the Greisen particle generator [22]. For each $e^{+} / e^{-}$generated, it computes and adds all the individual field contributions at any given point (even at heights near the shower maximum). Combining NEC's simulations for the VEL in the frequency domain 30-80 $\mathrm{MHz}$ with the electric field generated by SELFAS for various primary directions ${ }^{5}$ we calculated the voltage response model for the RF system $V_{e w / n s}(\theta, \phi, f)$. An example is shown in figure 6.

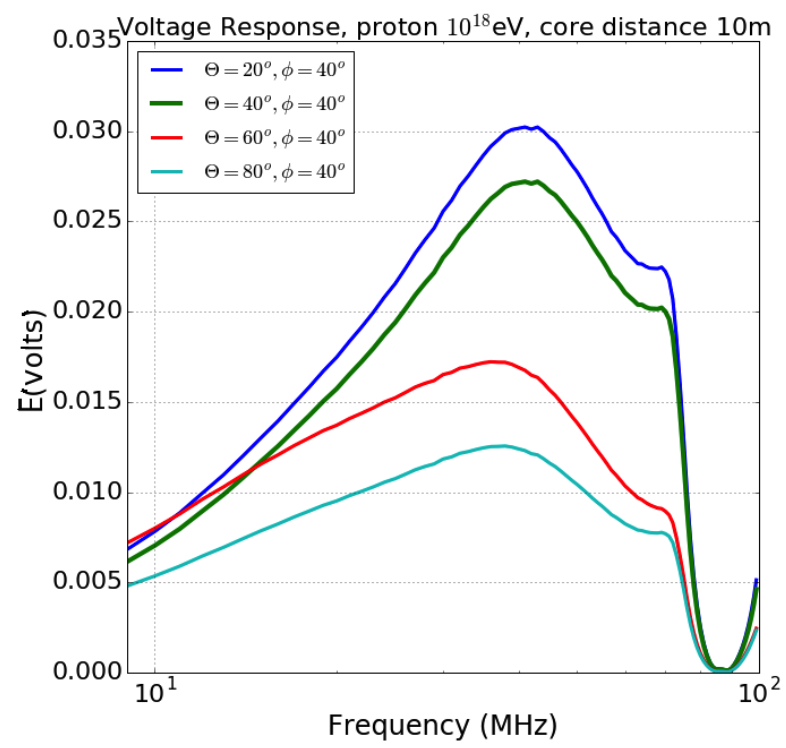

Figure 6. Butterfly Antenna voltage response model for EW pole for a shower generated by SELFAS. The proton's energy is $10^{18} \mathrm{eV}$ and the distance between the shower core and the antenna is $10 \mathrm{~m}$. The arrival directions are $\theta=20^{\circ}, \phi=40^{\circ}$ (blue), $\theta=40^{\circ}, \phi=40^{\circ}$ (green), $\theta=60^{\circ}, \phi=40^{\circ}$ (red), $\theta=80^{\circ}, \phi=40^{\circ}$ (light blue).

\subsection{Estimation of the Primary Arrival Direction}

The estimation of the primary arrival direction is performed by comparing the RF spectrum $V_{e w / n s}^{\text {spectrum }}(f)$ of the recorded signals with the $V_{e w / n s}(\theta, \phi, f)$ response model described in the previous subsection. The $V_{e w / n s}(\theta, \phi, f)$ response is evaluated for a large number of $(\theta, \phi)$ pairs (i.e. for $0 \leq \theta \leq 90,0 \leq \phi \leq 360$ with a step of one degree) and many different frequency values in the region

\footnotetext{
${ }^{5}$ Only the angular direction is used while the shower core, the primary energy and primary type were fixed to $10 \mathrm{~m}, 10^{18} \mathrm{eV}$ and proton respectively.
} 
$0-100 \mathrm{MHz}$. Then the shower direction is estimated by the minimization of the following $\chi^{2}$ value:

$$
\chi^{2}=\sum_{f=30-80 M H z}\left(\alpha \cdot V_{e w / n s}(\theta, \phi, f)-V_{e w / n s}^{\text {spectrum }}(f)\right)^{2}
$$

In equation 6 the summation is performed over the frequency values (a step of $1 \mathrm{MHz}$ was used) and for both poles of the antenna while the parameter $\alpha$ is a factor to scale the $V_{e w / n s}(\theta, \phi, f)$ for any energy, primary type and core distance.

The procedure for the evaluation of the RF spectrum $V_{e w / n s}^{\text {spectrum }}(f)$ of the recorded signals is the following. First both the EW and NS signals are filtered keeping frequencies in the range $30-80 \mathrm{MHz}$. Then the filtered signals are subjected to Hilbert transformation $\mathcal{H}$ (or envelop) being the convolution of the signal $V(t)$ with the function $\frac{1}{\pi t}$,

$$
\mathcal{H}(V(t))=\frac{1}{\pi} \int_{-\infty}^{+\infty} \frac{V(\tau)}{t-\tau} d \tau
$$

The Hilbert envelop is used to make the spectrum smoother removing possible remaining sources of noise (with frequencies near the cosmic event $45-55 \mathrm{MHz}$ ) and negative frequency components. Finally, the $V_{e w / n s}^{\text {spectrum }}(f)$ is computed by the Fourier transformation of $\mathcal{H}(V(t))$ for each antenna pole.
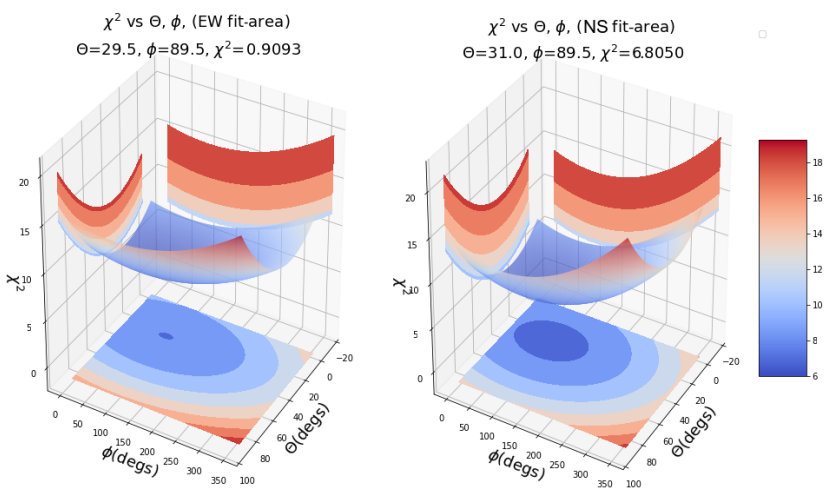

Figure 7. $\chi^{2}$ as a function of $\theta, \phi$ for EW and NS polarization for a cosmic event. The direction of this event using the particle detetor data is estimated to be $\theta=30^{\circ}$ and $\phi=93^{\circ}$.

An example of the minimization procedure is shown in figures 7 and 8 . The $\chi^{2}$ as a function of $\theta, \phi$ is evaluated by the corresponding values $V_{e w / n s}(\theta, \phi, f)$ and the $V_{e w / n s}^{\text {spectrum }}(f)$ (figure 7). The response model $V_{e w / n s}(\hat{\theta}, \hat{\phi}, f)$ where $\hat{\theta}$ and $\hat{\phi}$ denote the estimated values from the minimization is shown in figure 8 in comparison with the response RF spectrum $V_{e w / n s}^{\text {spectrum }}(f)$.

The estimated zenith and azimuth angles of the shower axis using the VRM can be compared with the corresponding values estimated from the particle detectors. The distribution of the difference on the zenith angle and on the azimuth angle are shown in figures 9 and 10 respectively. Both distributions are well described by Gaussian functions centered near zero. The corresponding sigmas of the Gaussian function are $\sigma_{\Delta \theta}=6.0^{\circ}$ and $\sigma_{\Delta \phi}=9.6^{\circ}$.

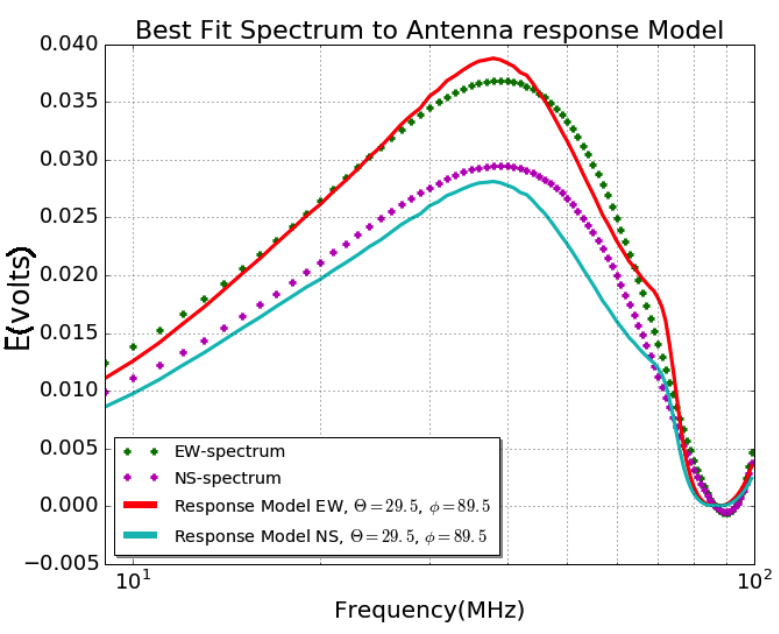

Figure 8. The comparison between the response model $V_{e w / n s}(\hat{\theta}, \hat{\phi}, f)$ and the $V_{e w / n s}^{\text {spectrum }}(f)$ for a cosmic event. $\hat{\theta}$ and $\hat{\phi}$ denote the estimated values from the minimization procedure shown in figure $7\left(\hat{\theta}=29.5^{\circ}, \hat{\phi}=89.5^{\circ}\right)$. The green (magenta) crosses is the EW (NS) spectrum and the red (light blue) line the EW (NS) VRM.

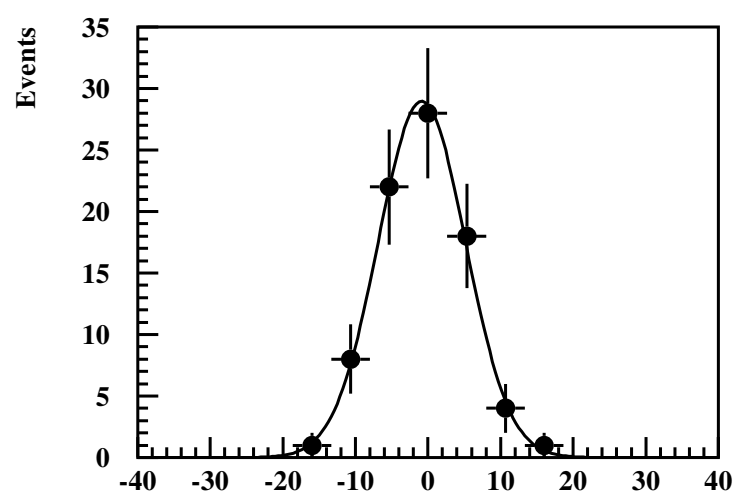

Figure 9. The distribution of $\Delta \theta$ between the zenith angle estimated using the VRM and the corresponding angle estimated using the particle detector data. The distribution is fitted with Gaussian function of sigma equal to 6 degrees.

\section{Conclusions}

Previous results from the Astroneu array have shown that using particle detectors to trigger the antennas, the RF signature of EAS can be distinguished in a noisy city environment. More recent studies have highlighted that the RF signal timing at the peak of the EW signal is compatible with the expectation using the HDM pulses timing and the plane particle front approximation while the electric field strength measurement agrees with the MC expectations. In this study double station coincidence events between stations $\mathrm{A}$ and $\mathrm{B}$ of the Astroneu array were used to examine the potential of a single RF antenna in estimating the angular direction of extensive air showers. Using the strength of the EW signal and the ToT values of the HDMs a clean 


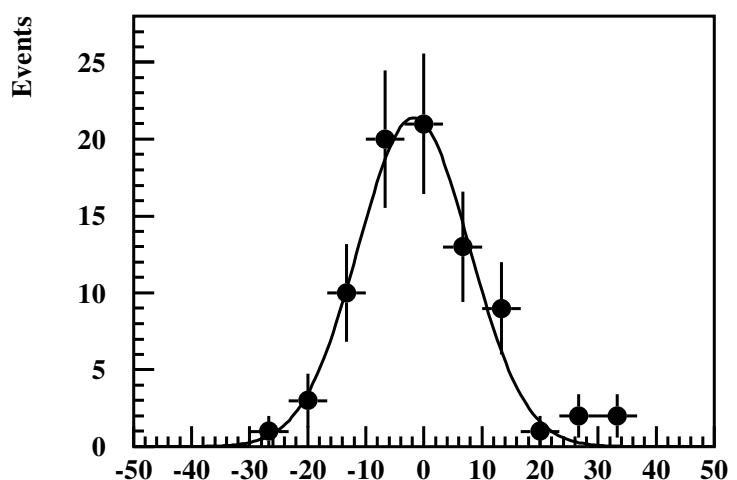

Figure 10. The distribution of $\Delta \phi$ between the azimuth angle estimated using the VRM and the corresponding angle estimated using the particle detector data. The distribution is fitted with Gaussian function of sigma equal to 9.6 degrees.

sample of very high energy showers were selected for a running period of about one year and a half. This sample was analyzed in order to obtain the voltage response spectrum for frequencies between 30 and $80 \mathrm{MHz}$. The spectrum was compared on an event by event basis with a voltage response model evaluated using the vector effective length of the antennas and MC simulation. It was found that the estimated values of the zenith and azimuth angle direction are in very good agreement with the estimations using the timing of the particle detectors. The analysis is still on going in order to define more efficient selection criteria and signal processing techniques in the full data set which is a factor of 2 grater than the sample used in this analysis. In addition, to examine further the potential of the RF detection, more antennas have been installed in one of the stations. Specifically, since 2017 station-A is equipped with $4 \mathrm{RF}$ antennas receiving a common trigger from the HDM detectors. This will allow to study the correlation between RF signals corresponding to the same shower events.

Funding: This research was supported by the Hellenic Open University Grant No. $\Phi K$ 228: "Development of technological applications and experimental methods in Particle and Astroparticle Physics."

Acknowledgments: We would like to thank Codalema experiment and especially Dr. Lillian Martin and Dr.Didier Charrier for their precious support in this work. We would like also to thank Michael Petropoulos, George
Georgis and the technical staff of HOU for their support in operating the Astroneu array.

\section{References}

[1] D.Ardouin et al., Nucl. Instrum. Methods A, 555, 148163 (2005).

[2] P. Schellart, A. Nelles S. Buitink et al., Astron. Astrophys.,560, 98 (2013).

[3] Pierre Auger Collaboration,Nucl. Instrum. Meth. A 798, 172-213 (2015).

[4] Schröder F. G., Prog. Part. Nucl. Phys., Elsevier, 93, 1-68 (2017)

[5] Huege, T. Phys. Rep., Elsevier BV, 620, 1-52 (2016)

[6] O. Scholten K.Werner F. Rusydi., Astropart. Phys., 29, 94-103 (2008).

[7] G.A. Askaryan, Phys. JETP 14, 441 (1962).

[8] I. Manthos et al., arXiv:1702.05794 (2017), to be submitted.

[9] A. Leisos et al., submitted to Universe (2018).

[10] D. Charrier, Nucl. Instrum. Methods Phys. Res., Sect. A 662, 142-145 (2012).

[11] A. Leisos et al., submitted to Universe (2018).

[12] T. Avgitas et al, arXiv:1702.04902 (2017), to be submitted.

[13] T. Avgitas et al., arXiv:1801.04768 (2017), to be submitted.

[14] D. Charrier, IEEE Antennas and Propagation Society International Symposium, 4485-4488 (2007).

[15] S. Hansen et al, IEEE Transactions on Nuclear Science, V.51, 926-930 (2004)

[16] O. Ravel, Nucl. Instrum. Methods Phys. Res., Sect., A 662, 89-94 (2012).

[17] C.A. Balanis, Antenna Theory- 3rd Edition, (Wiley, 2010).

[18] G. Burke and A. Poggio, Numerical Electromagnetics Code (NEC) method of moments, parts I, II, III, tech. rep., (Lawrence Livermore National Laboratory, NEC3, 1983).

[19] J. D. Kraus and R. J. Marhefka, Antennas, (McGrawHill, 2003).

[20] P. Abreu for the Pierre Auger Collaboration, Nucl. Instrum. Methods Phys. Res., B 521, 65-72 (2013).

[21] V. Marin and B. Revenu, Astropart. Phys. 35, 733 (2012)

[22] B. Rossi et K. Greisen, Reviews of Modern Physics 13, 240-309 (1941). 